\title{
Unified European Gravity Reference Network 2002 (UEGN02) - Status 2004
}

\author{
G. Boedecker
}

Bavarian Academy of Sciences and Humanities / BEK, München, Germany. Fax +498923031100

boe@bek.badw-muenchen.de

O. Francis .

European Centre for Geodynamics and Seismology / University of Luxembourg,Luxembourg, Fax +35233

148788 , olivier@ecgs.lu

\author{
A. Kenyeres \\ FOMI Satellite Geodetic Observatory, Penc, Hungary; Fax +3627 374982, kenyeres@sgo.fomi.hu
}

\begin{abstract}
The last previous realisation of the Unified European Gravity Reference Network was completed in 1994 (UEGN94) covering 11 West European countries. Since that time significant work has been done e.g. in the frame of the UNIGRACE project that focused on the establishment of absolute gravity stations in Central and East European countries.

The past European Subcommission of the IGGC recommended to continue with the unification of the gravity reference networks at the continental level. During the IAG2001 Scientific Assembly in Budapest, it was decided to establish a new continental gravimetric reference network.

The total UEGN2002 network area covers 25 countries, the number of stations expected was around 1000 , but has already exeeded this number. The data processing starts from raw absolute and relative gravity observations. The tidal corrections are computed by the ECGS in Luxembourg in a uniform manner and based on state-of-the-art models.
\end{abstract}

At the current status (2004) the work is focused on 12 countries with 404 absolute and 33000 relative gravity observations at some 1500 stations. Main problems are data bugs. Hence, appropriate tools had to be developed.

The paper presents details of the adjustment, earth tide corrections as well as analyses of the data and adjusted gravity values. After this partial UEGN02 adjustment, the remaining countries that also showed their interest to participate are again invited to submit data.

Keywords. Reference network, gravity reference

\section{Introduction}

\subsection{Role of Gravity Reference Networks}

Because bulk field gravimetry still is carried out by relative spring gravimeters, it is necessary to link these to an absolute reference. Decades ago, only a few absolute observations worldwide provided the basis for extended relative gravity reference networks. Today, as absolute gravity meters become more easily available, they become more fieldworthy and portable, also their number is increasing. Consequently, the number of absolute stations is increasing and the contribution of relative meters to reference networks is decreasing. In each case where a new national reference or an extended field campaign is envisaged, the benefit-cost-relation will lead to a new optimum design appropriate to the specific situation, see e.g. Boedecker (2002). Relative observations are not only important for reference networks, their use in combined absolute / relative networks will also benefit in their calibration.

\subsection{European cooperation / Preceding works}

After the old global gravity reference network IGSN71 (Morelli et al. 1971) proved to be not a sufficiently accurate basis for new gravity observations any more, many countries designed and observed national networks. In 1994, an attempt was made on behalf of the IGC Subcommission Western Europe to unify a number of European national networks (Boedecker, Marson, Wenzel 1994) and named UEGN94 ('Unified European Gravity Networks 1994'). The network covered 11 countries 
comprising 499 stations with 123 absolute and 14532 relative gravity observations; see fig. 1 .

From 1998 to 2002 the project UNIGRACE ('Uni-

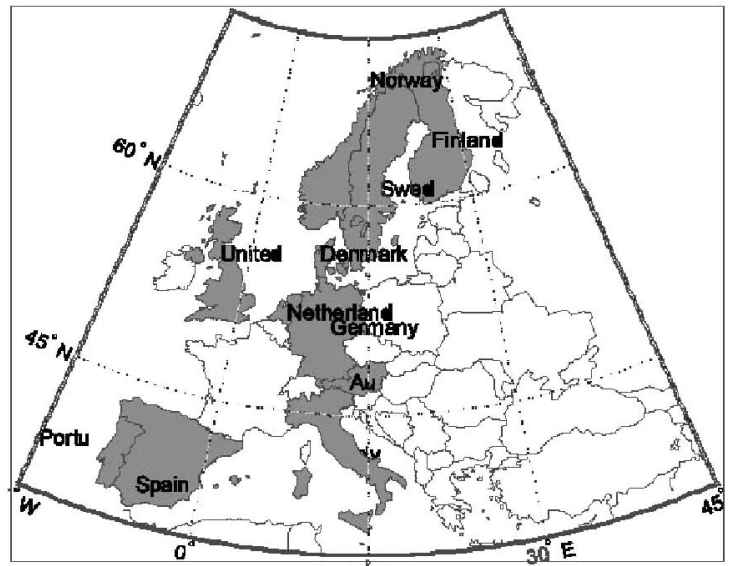

Figure 1: UEGN94

fication of Gravity Systems in Central and Eastern Europe') was carried out, coordinated by the German BKG ("Bundesamt für Kartographie und Geodäsie') with contractors from all participating countries, see fig. 2. See Reinhart, Richter, Wilmes 1998. In the framework of that project, absolute observations at 19 stations in 12 countries in central and eastern Europe have been observed and were made available to the national survey agencies and to the UEGN project.

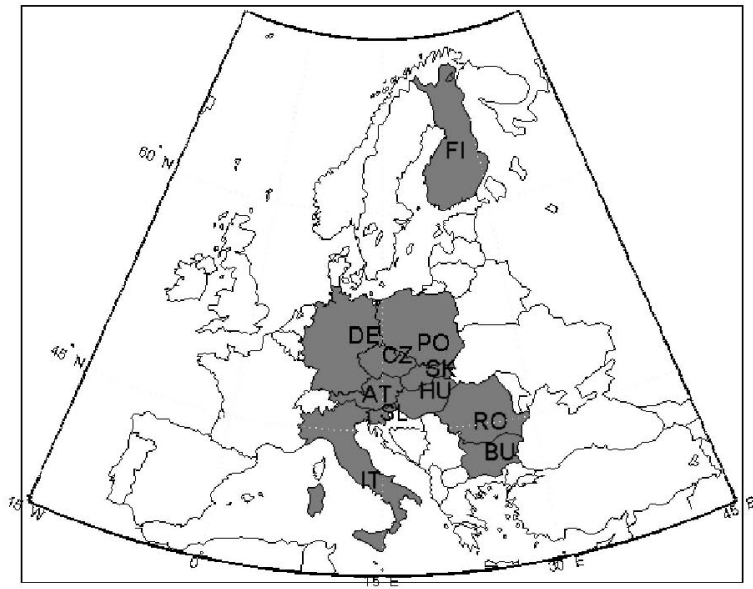

Figure 2: UNIGRACE

These works for a unification of European gravity reference networks were continued by the UEGN02. A first meeting to agree on the guidelines took place on May 13-14, 2001, in Vienna.

The work was delayed because of problems with details of the ties between absolute and relative observation - a key problem for the correct gravity level of the network.

Some further delay is caused by incorrect data: As a matter of fact, this is a bigger problem than in other much larger data sets, because in this case we are dealing with (currently) 33000 observations each of which has its own evolution and is not the result of one unique process as is the case in other type of geodetc observations like GPS observation series or similar. For this reason, some software tools had to be developed in order to make the observation series and procedures more transparent. The current status of the UEGN02 activities is illustrated in figure 3 :

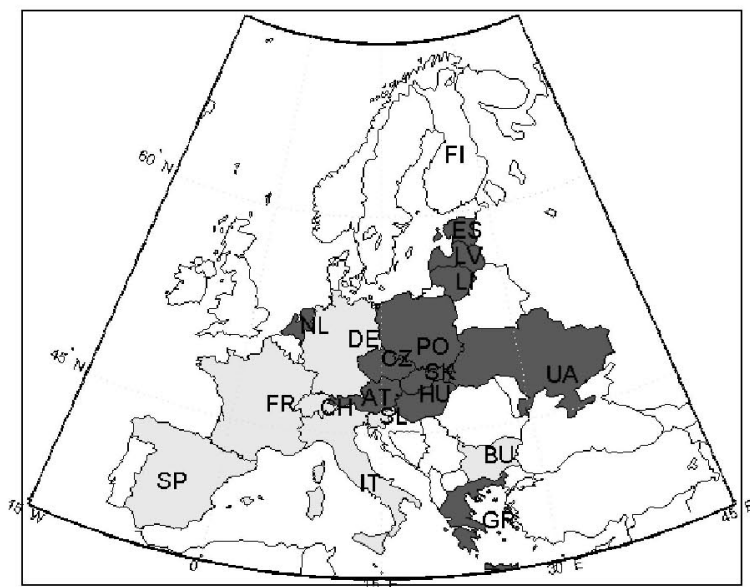

Figure 3: UEGN02: Activity status 08/04 dark: adjustment computation, data cleaning medium: some data available light: data envisaged

\section{Data collection}

The data were collected in four types of formatted files: station data, absolute observations, relative observations. Some national agencies do not agree to have their data published. For this reason, no original data set will be transferred without permission of the original owner.

\section{Tidal reduction}

All the raw observations of the relative gravity meters have been corrected for the body Earth tides and ocean loading and attraction effect using stateofthe art models. The tidal parameters for the body Earth tides come from the Dehant-Defraigne-Wahr (1999) model for an inelastic non-hydrostatic Earth (Dehant et al., 1999) including a delta factor of 1.16 for the long periods tides. The body tides prediction 
is computed with the etgtab software written by Wenzel (http://www-geod.kugi.kyotou.ac.jp/iagetc/etcdat/etgtab/etgtab.txt

On the other hand, the ocean loading and attraction parameters were calculated using the ocean tides model CSR3.0 and the Green's function of the PREM with the Load89 software (Francis O. and P. Mazzega, 1993). The oceanic loading tidal prediction is then calculated using the ocean loading parameters in a separate program. The tidal correction is the sum of the contributions: body Earth tides and oceanic loading and attraction gravity effect.

The data from the absolute gravimeters were provided already corrected for tides. It means that the tides correction for these data could be slightly different. It was outside the scope of this work to reprocess all the absolute gravity data from the raw observations. ).

\section{Adjustment model}

The adjustment model is identical to the adjustment of UEGN94. Absolute gravity observations are immediate observations of station gravity.

Absolute gravity observations :

$v_{i}=g_{j}-\widetilde{g}_{i}$

$v_{i}$ residual of observation $\mathrm{i}$

$g_{j}$ unknown station gravity

$\widetilde{g}_{i}$ observed gravity incl. tidal reduction

Relative gravity meter obervations are taken as a series of consecutive observations within one day by one gravity meter. A series is modeled by station gravity values, an offset (per series), linear drift of the readings with time (per series) and a linear calibration factor for the entire set of observations of the meter. Relative observations are the raw observations - corrected for e.g.periodic errors, if necessary or available, converted by the manufacturers calibration table to care for nonlinear components, and reduced by tidal effects (see below).

\begin{tabular}{|ll|}
\hline Relative gravity observation : \\
$v_{i}=g_{j}+o_{l}+z_{i} f_{g}+t_{i} d_{l}-r_{i}$ \\
$v_{i} \quad$ residual of observation $\mathrm{i}$ \\
$g_{j} \quad$ unknown gravity of station $\mathrm{j}$ \\
$o_{l} \quad$ orientation of series 1 \\
$z_{i} \quad$ raw gravimeter reading $\mathrm{i}$ \\
$f_{g} \quad$ linear calibration factor of gravimeter $\mathrm{g}$ \\
$t_{i} \quad$ time of observation i \\
$d_{l} \quad$ linear drift for series l \\
$r_{i}$ & gravimeter reading i incl. tides \& pre - calib. \\
\hline
\end{tabular}

The intention was to use the original observations at definition height of the absolute observation and to connect to all other stations including the immediate ground marker by relative observations. This would have avoided introducing prior 'gradient corrections' etc. However, frequently the original relative gravity observations were not carried out at the height of the absolute observations; hence, the ties between absolute observations and the network is a mix of immediate relative observations and intermediate use of gradients.

To account for the variations in accuracy of the absolute observations, the internal error variances estimations were introduced, as communicated by the agencies providing the data.

The weights of the relative observations were assumed constant within one series and the series weights were estimated from an iterative variance component estimation.

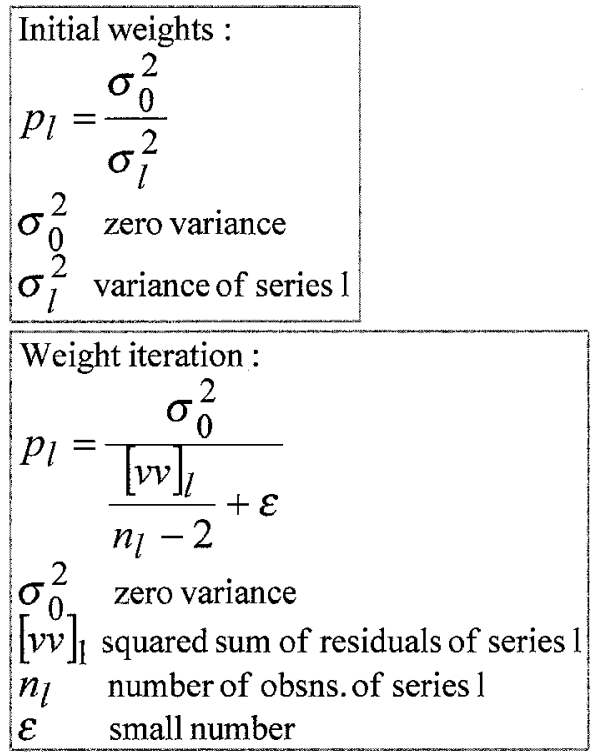

\section{Execution of adjustment}

The adjustment programme was developed in MATLAB. The challenges of this work are in the data organization etc. E.g., relative observation series have to be identified from the contiguous observation set, station data checked; series of only two observations do not provide network information and are removed.

The data were processed to a certain point, then metadatafiles were transferred to ECS Luxembourg (O. Francis) for tidal reduction. After this, the processing continued. Variants of the adjustment are based on the same data set, hence the tidal 
reduction computation was necessary only once so far.

Because of the restrictions of this paper format, it is not possible to provide a full and detailed image of the screen for the practical work on the network. Rather, a sequence of cutout figures tries to provide at least a rough impression, see appendix.

The network figures (see appendix) show e.g. normal stations, absolute stations, stations with observations by parties from other countries, station names, station gravity standard deviation estimates, absolute and relative residuals above some threshold (arrows), observation series with residuals above some threshold (red dotted line).

The graphical user interface is not just a nice toy, but it is essential because otherwise a clarification of bugs in the large data set of individual observations would not be possible.

A standard programme run including all original data file input and output, graphics, parameter estimation for about 10.000 parameters, iterative covariance component estimates in six iterations, but without tidal reduction and inversion of the normals takes less than 1 minute on a normal PC under Windows.

The inversion for computing the standard deviations of the parameters takes between half a minute to several minutes depending on the quality of the normal matrix.

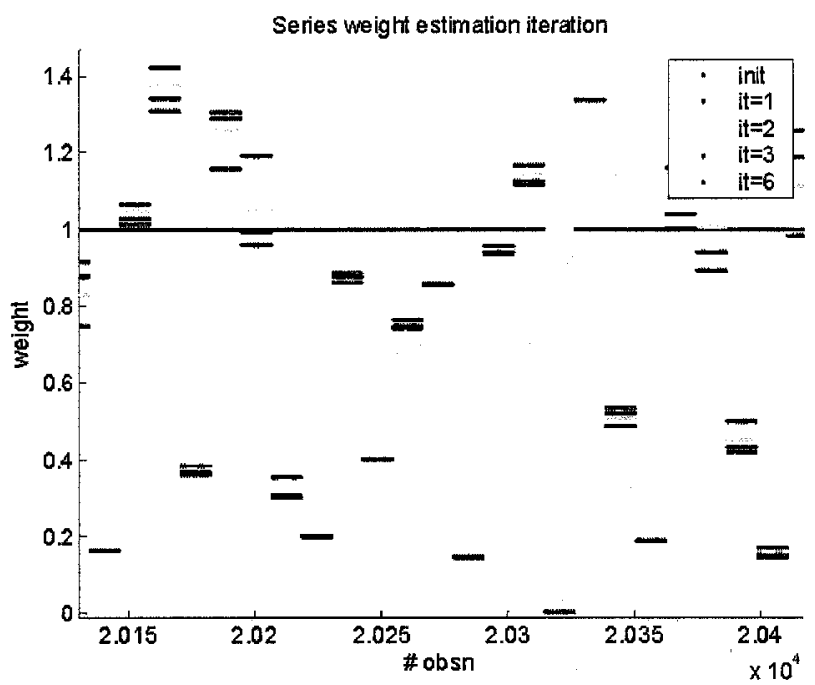

Figure 4: Iterative weight estimation

Further explanation see text

The weight estimation for relative observations starts with unit weight (black in the sample figure 4), except the series is downweighted to zero as a result of a preceding bug analysis (see figure). The iteration subsequently yields the weights depicted above.

As can be recognized, the convergence is quite stable and fast.

The key numbers of the sample run are given below. The histogram of residuals shows that $50 \%$ of the residuals are less than $0.007 \mathrm{mGal}$.

Absolute obsns: 405

Relative obsns: 33092

Rel. gravimeters 44

Series $\quad 3989$

Stations: $\quad 1446$

Parameters: $\quad 9445$

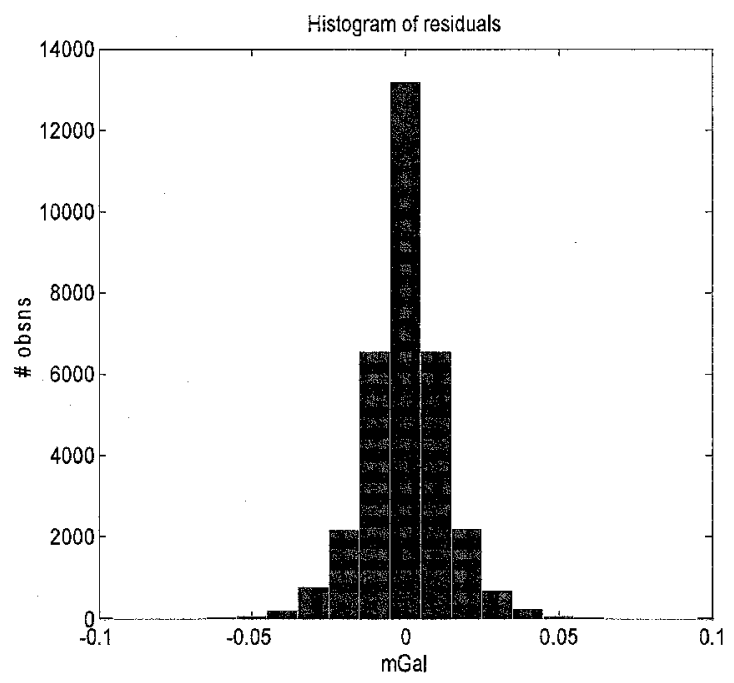

Figure 5: Histogram of residuals

\section{Conclusions and outlook}

- A subnet of the UEGN02 has successfully been processed

- The national network structures are very diverse

- Observation data bugs are a major problem

- This project completes the UNIGRACE objectives

- The accuracy is satisfactory

- The benefits are very manifold

- All European countries are called to participate

- Some of the countries contributing already are called to deliberate to submit more complete data in order to have greater benefit 
- It is envisaged to complete the project in 2005

- The further procedure, e.g. publication (also stations ?) has to be agreed by the participating countries

\section{Acknowledgements}

This work has been made possible through the contributions of many colleagues in institutes and agencies in the participating countries, Austria, Czech Republic, Croatia, Estonia, Finland, France, Germany, Greece, Hungary, Italy, Latvia, Lithuania, Netherlands, Poland, Slovakia, Slovenia, Spain, Switzerland, Ukraine. Others offered there active participation in computations such as Völgyesi and Csapo from Hungary, Simek from Czech Republic and Klobusiak from Slovakia; these offers are appreciated.

\section{References}

Boedecker, G.: World gravity standards - present status and future challenges. Metrologia 2002, 39 no. $5,429-434$

Boedecker, G.; Marson, I.; Wenzel, H.-G. : The Adjustment of the Unified European Gravity Network 1994 (UEGN 94). In: Sünkel, H. / Marson, I. (eds.): Gravity and Geoid. Joint IGC/ICG Symposium Graz 1994. IAG Symposium No. 113, Springer 1995, 82-91

Dehant, V., P. Defraigne and J. M. Wahr, Tides for a convective Erath, Journal of Geophysical Research, 104, 1035-1058, 1999.

Francis O. and Mazzega P., Global charts of ocean tide loading effects, Journal of Geophysical Research, 95(C7), 11411-11424, 1990.

Morelli, C., Gantor, C., Honkasalo, T., McConnell, R.K., Tanner, J.G., Szabo, B., Votila, V. \& Whalen, C.T.-1971- The International Gravity Standardisation Net. Pub. Spec. no. 4 du Bulletin Géodésique., 1974

Reinhart, E.; Richter, B.; Wilmes, H.: "UNIGRACE - Ein Projekt zur Vereinheitlichung der Schwere-Referenzsysteme in Zentral-Europa" Mitteilungen des Bundesamtes für Kartographie und Geodäsie, Band1, Frankfurt a. M. 1998;

see also:

INCO: International Scientific Cooperation Projects (1998-2002) Contract Number: ERBIC15CT970805 FINAL REPORT

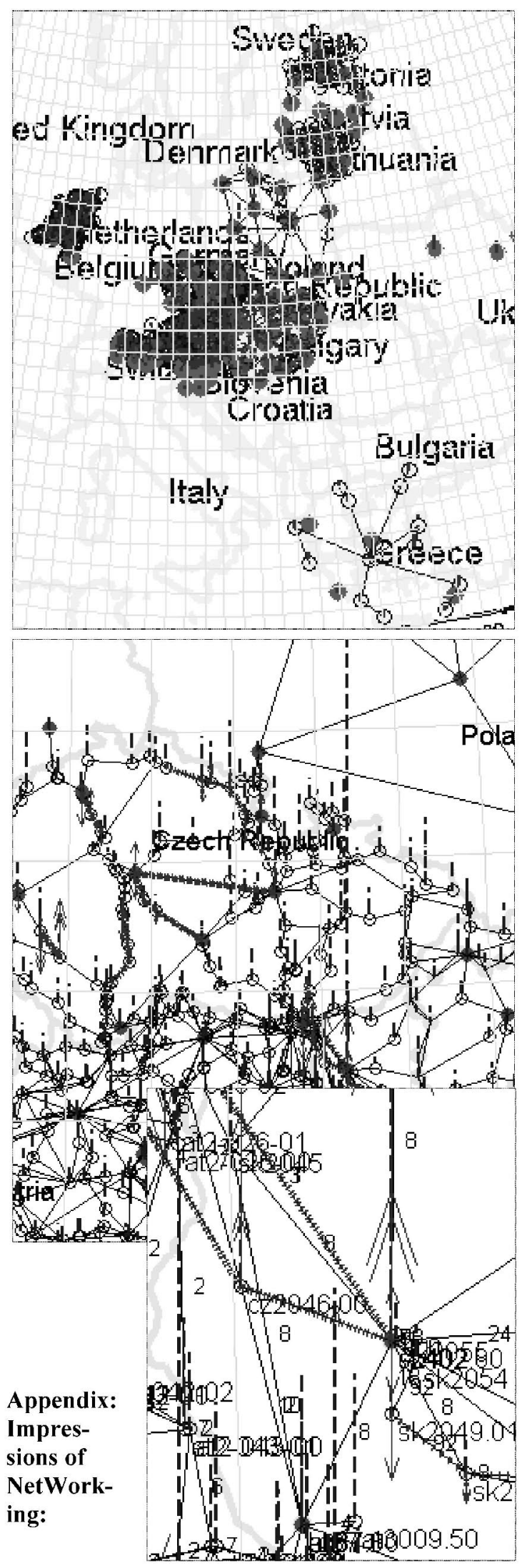


Appendix:

Sample output

Parameters not solved for:

Station Gravity:

794 gr299RAFI

797 gr309KYMH

\{

Observations per

instrument: \# instr, \# obsns

$\begin{array}{ccc}1 & -900.002 & 1008 \\ 2 & -625.001 & 110 \\ 3 & -90.002 & 178 \\ 4 & 3.004 & 302 \\ 5 & 4.001 & 26 \\ 6 & 51.002 & 1934\end{array}$

UEGN results

$2004 \quad 8191912$

Stations files read:

D:IUEGN02lat lstations_at_03.txt4

D:IUEGN02lczlstations_cz_02.txt4

Absolute observations files read:

D:UUEGN02latlabsobs_at_03.txt 5

D:IUEGN02lczlabsobs_cz_02.txt 5

Relative observations files read:

D:IUEGN02lattrelobs at $4 b . t \times t \quad 5$

D:IUEGN02lcz।relobs_cz_2a.txt 5

Stations with identical coordinates 3D :

at0-059-20 atA-059-20

cz107.10 hu107.10

cz107.10 sk107.10

cz108.00 hu108

Parameters

names (seq: grav, lincal, orient, drift), adjusted values, mse:

at0-021-00 $980867.380 \quad 0.002$

at0-021-01 $980868.928 \quad 0.003$

at0-050-00 $980741.267 \quad 0.002$

at0-058-20 $980842.055 \quad 0.003$

$\begin{array}{lll}180.041 & -0.089 & 0.000\end{array}$

$\begin{array}{lll}191.001 & 6.083 & 0.001\end{array}$

$\begin{array}{lll}192.001 & -3.727 & 0.001\end{array}$

$\begin{array}{lll}192.05 & 2.749 & 0.006\end{array}$

$193.001 \quad-23.301 \quad 0.001$

Observations per stations: / \# rel obsns, \# abs obsns

at0-021-00 $65 \quad 0$

at0-021-01 $17 \quad 0$

hu87 70

hu88 $\quad 0 \quad 2$

hu88.0 $54 \quad 2$

hu89 01

..

Absolute outliers:

hu88 $930826980765.513000 \quad 0.048$ AXIS-FG5-107 0.002 Gschwind

hu88 $971213980765.610000-0.049$ JILAG-6 0.002 Ruess

Bad series:

Bad series:

mse, maxr: 0.0572300 .060223

(StatName, date, time, instr, raw, obs(inc cal/tid), tides, resid, status)

at1I-SCHLb $0109291837 \quad-900.002 \quad 119.817 \quad 134.397-0.056+0.0310$

at2-171-20 $0109291946 \quad-900.002 \quad 17.798 \quad 19.951 \quad-0.032 \quad-0.0130$

at2-171-21 $0109292012 \quad-900.002 \quad 42.309 \quad 47.471 \quad-0.023 \quad-0.0600$

at1I-SCHLb $0109292114 \quad-900.002 \quad 119.777 \quad 134.402-0.006+0.0420$ 